\title{
Modifying the Interface Edge to Control the Electrical Transport Properties of Nanocontacts to Nanowires
}

\author{
Alex M. Lord, *†® Quentin M. Ramasse," Despoina M. Kepaptsoglou," Jonathan E. Evans, \\ Philip R. Davies, ${ }^{\perp}$ Michael B. Ward, ${ }^{\#}$ and Steve P. Wilks ${ }^{\S}$ \\ ${ }^{\dagger}$ Centre for NanoHealth, College of Engineering, ${ }^{\ddagger}$ Centre for NanoHealth, College of Science, and ${ }^{\S}$ Multidisciplinary \\ Nanotechnology Centre, Department of Physics, College of Science, University of Swansea, Singleton Park SA2 8PP, United \\ Kingdom \\ "SuperSTEM Laboratory, SFTC Daresbury Campus, Keckwick Lane, Daresbury WA4 4AD, United Kingdom \\ ${ }^{\perp}$ Cardiff Catalysis Institute, School of Chemistry, Cardiff University, Park Place, Cardiff CF10 3AT, United Kingdom \\ \#Institute for Materials Research, University of Leeds, Leeds, LS2 9JT United Kingdom
}

\section{Supporting Information}

\begin{abstract}
Selecting the electrical properties of nanomaterials is essential if their potential as manufacturable devices is to be reached. Here, we show that the addition or removal of native semiconductor material at the edge of a nanocontact can be used to determine the electrical transport properties of metal-nanowire interfaces. While the transport properties of as-grown $\mathrm{Au}$ nanocatalyst contacts to semiconductor nanowires are well-studied, there are few techniques that have been explored to modify the
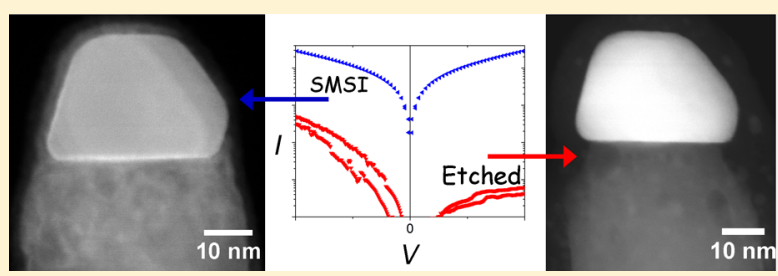
electrical behavior. In this work, we use an iterative analytical process that directly correlates multiprobe transport measurements with subsequent aberration-corrected scanning transmission electron microscopy to study the effects of chemical processes that create structural changes at the contact interface edge. A strong metal-support interaction that encapsulates the Au nanocontacts over time, adding $\mathrm{ZnO}$ material to the edge region, gives rise to ohmic transport behavior due to the enhanced quantummechanical tunneling path. Removal of the extraneous material at the $\mathrm{Au}$-nanowire interface eliminates the edge-tunneling path, producing a range of transport behavior that is dependent on the final interface quality. These results demonstrate chemically driven processes that can be factored into nanowire-device design to select the final properties.

KEYWORDS: Nanowires, electrical contacts, tunneling edge effect, aberration-corrected scanning transmission electron microscopy, strong metal-support interaction, $\mathrm{ZnO}$
\end{abstract}

$\mathrm{M}$ anufactured devices are expected to function with predetermined electrical properties throughout their lifetime, and this imposes the same requirement on any nanomaterial-based components. Recent advances in experimental and analytical techniques have allowed researchers to investigate chemically and structurally driven modifications that determine the electrical properties of $1 \mathrm{D}$ nanostructures. Remarkable effects have been reported, such as the modification of nanowires with core-shell growth ${ }^{1-3}$ and the migration of the Au catalyst materials through the bulk of $\mathrm{Ge}$ and GaAs nanowires ${ }^{4,5}$ and away from the tips of Si nanowires to control the $\mathrm{Au}$ shape. ${ }^{6}$

The Au nanoparticle catalyst that promotes the growth of many nanowires holds great promise as a high-quality and ready-made electrical nanocontact at the tips of the $1 \mathrm{D}$ structures, removing any need for postgrowth processing to create complete devices. ${ }^{6}$ Recent work on Au nanocontacts to $\mathrm{ZnO}$ nanowires has shown that the transport properties can be switched from ohmic to Schottky as a result of quantummechanical edge-tunneling effects by selecting a nanocontact that is comparable in diameter to the nanowire. ${ }^{7}$ In structurally controlled nanocontacts to planar surfaces, a change in electrical transport properties has been reported by varying the atomic structure of the interface. ${ }^{8-10}$ Currently, efforts to control the transport properties of nanowire catalyst contacts are limited to changes in the interface dipole, achieved by metal alloying or heterostructure formation, and size effects that modulate carrier transport. ${ }^{7,11,12}$ However, a number of techniques exist that could be exploited to control the electrical characteristics of the Au contacts such as etching, ${ }^{13}$ nanowire surface architecture modulation during growth, ${ }^{14}$ or the addition of nanowire shell materials. ${ }^{1-3}$ Furthermore, there has been little detailed study of the transport behavior of nanowires with $\mathrm{Au}$ contacts in relation to changes in the atomic-scale structure and chemistry of the interface region. In this work, we investigate nanoscale modifications to the material at the interface edge of the $\mathrm{Au}-\mathrm{ZnO}$ nanowire interfaces and directly relate the transport properties to the

Received: September 2, 2016

Revised: November 26, 2016

Published: December 21, 2016 
structure and form of the interface using aberration-corrected scanning transmission electron microscopy (ac-STEM). The $\mathrm{Au}-\mathrm{ZnO}$ nanowire system is an ideal candidate for these investigations because of the abrupt and prototypical nature of the interface. Chemical and structural modifications of the nearinterface semiconductor surface reveal that the state of the $\mathrm{ZnO}$ at the contact edge modulates the dominant tunneling transport mechanism. This is initially demonstrated with aged nanowires that exhibit a strong metal-support interaction (SMSI) that encapsulates the Au contacts, adding $\mathrm{ZnO}$ material to the edge region that produces ohmic transport behavior as opposed to rectifying behavior when the nanowires are in the initial nonaged state. ${ }^{7}$ Further modifications to the interface edge are performed by etching away the $\mathrm{ZnO}$ material that provides the edge tunneling path, producing a range of transport behavior that is dominated by the nature of the central interface.

The overriding conclusion of the work is that elementary modifications of the $\mathrm{ZnO}$ surface near the contact can provide a practical strategy with which to control the electrical transport properties. The demonstrated effects suggest interesting possibilities for transient electronics and show that engineering the nanomaterial contact properties can be achieved through simple post-growth treatment. ${ }^{11,15}$

Results and Discussion. Electrical Transport Properties of Modified Au Nanocontacts to ZnO Nanowires. The addition of native $\mathrm{ZnO}$ material to the contact edge region was achieved by chronologically aging $\mathrm{ZnO}$ nanowires grown by a metal-catalyzed vapor-phase technique ${ }^{16}$ for $\sim 3$ years in ambient laboratory conditions. The nanowires were then mechanically transferred to a standard copper four-post focused ion-beam transmission electron microscope (TEM) lift-out grid without any further treatment. The grids were screened in a conventional TEM to locate overhanging nanowires that had the $\mathrm{Au}-\mathrm{ZnO}$ interface close to parallel with the electron beam and to capture the initial state of the nanowires (Figure S1) before the first iteration of electrical transport measurements. The experimental (eSTEM) procedure described here initially employed an ultrahigh-vacuum (UHV) multiprobe scanning tunneling microscopy (STM) instrument with in situ scanning electron microscopy (SEM) capability to measure the transport behavior of the nanowires suspended on the grid. The multiprobe instrument allowed a tungsten probe to be maneuvered with nanometer precision, such that it could be accurately placed onto the $\mathrm{Au}$ tips of the $\mathrm{ZnO}$ nanowires (Figure 1a). Performing the electrical measurements in the UHV-STM instrument provided the advantage that the tungsten measurements probes could be thermally annealed in situ to remove oxides and contamination, ensuring the probe-to-Au contact was a low-resistance metal-on-metal contact (see the Experimental Methods section), and as such, the transport measurements revealed the properties of the $\mathrm{Au}-$ nanowire structures. ${ }^{7,17-19}$ After the electrical measurements were taken, the sample grid was then removed from the UHV multiprobe STM instrument, and the same nanowires were interrogated with ac-STEM imaging and electron energy-loss spectroscopy (EELS), providing access to the ultimate electron microscopy "on-axis" imaging resolution and spectroscopic signal.

Electrical in situ probing of nanoscale materials in the TEM has produced many important recent results, such as those on the lamella of ferroelectric materials at atomic resolution by Xiaoqing Pan's group at the University of California, Irvine, and
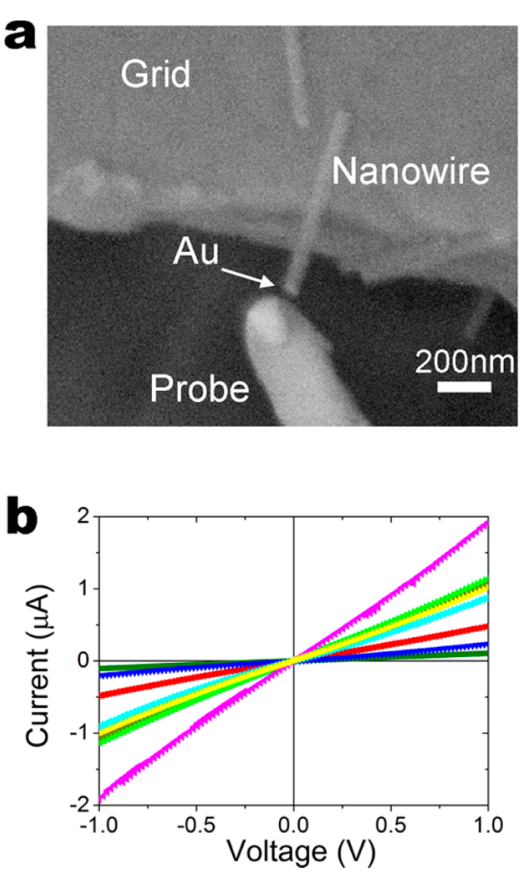

Figure 1. (a) In situ SEM image from the multiprobe UHV instrument used to measure the nanowires in the eSTEM cantilever configuration displaying the tungsten measurement probe, the nanowire, and $\mathrm{Au}$ nanocontact at the nanowire tip. (b) A graph showing I-V measurements of eight chronologically aged nanowires in the eSTEM configuration. A total of eight different examples are shown, all exhibiting similar ohmic-like transport behavior rather than the rectifying properties of nonaged nanowires.

on silicon nanowires by Frances Ross and co-workers at IBM. ${ }^{6,20-22}$ Dispersing nanomaterials on transmission electron microscopy grids has allowed in situ mechanical deformation of nanowires, ${ }^{23}$ while in situ biasing of nanowires has been used with electron holography to map electrostatic potential. ${ }^{24}$ In this work, application of the eSTEM approach was essential to ensure accurate electrical measurements with clean probes on multiple nanowires (35) to reveal the transport properties of the $\mathrm{Au}-\mathrm{ZnO}$ interfaces and also to allow ac-STEM analysis before and after ex situ modifications to the nanowires (a stepby-step description is provided in the Experimental Methods section). Additionally, the electrical properties of $\mathrm{ZnO}$ nanostructures are dominated by the surface, and the material is highly susceptible to any processing that is often necessary for in situ measurement devices.

The initial electrical measurements involved current-voltage $(I-V)$ sweeps between $\pm 1 \mathrm{~V}$ of the chronologically aged nanowires in the eSTEM configuration that showed a range of current magnitude from several tens of $\mathrm{nA}$ to a few $\mu \mathrm{A}$ and displayed linear ohmic-like $I-V$ characteristics for the 15 nanowires that were measured (Figure $1 \mathrm{~b}$ ). To confirm the measurements of ohmic transport behavior, vertical freestanding nanowires in the original chronologically aged growth array were measured in a two-probe configuration with the multiprobe UHV instrument (shown in Figure S2a,b with accompanying description). In the two-probe configuration, one probe connection forms a low-resistance ohmic contact to the nanowire shaft, and the second probe contacts the $\mathrm{Au}$ particle tip to perform $I-V$ measurements. ${ }^{7,18,25}$ The eight chronologically aged nanowires measured all showed nearlinear $I-V$ characteristics (Figure S2c), which is in agreement 

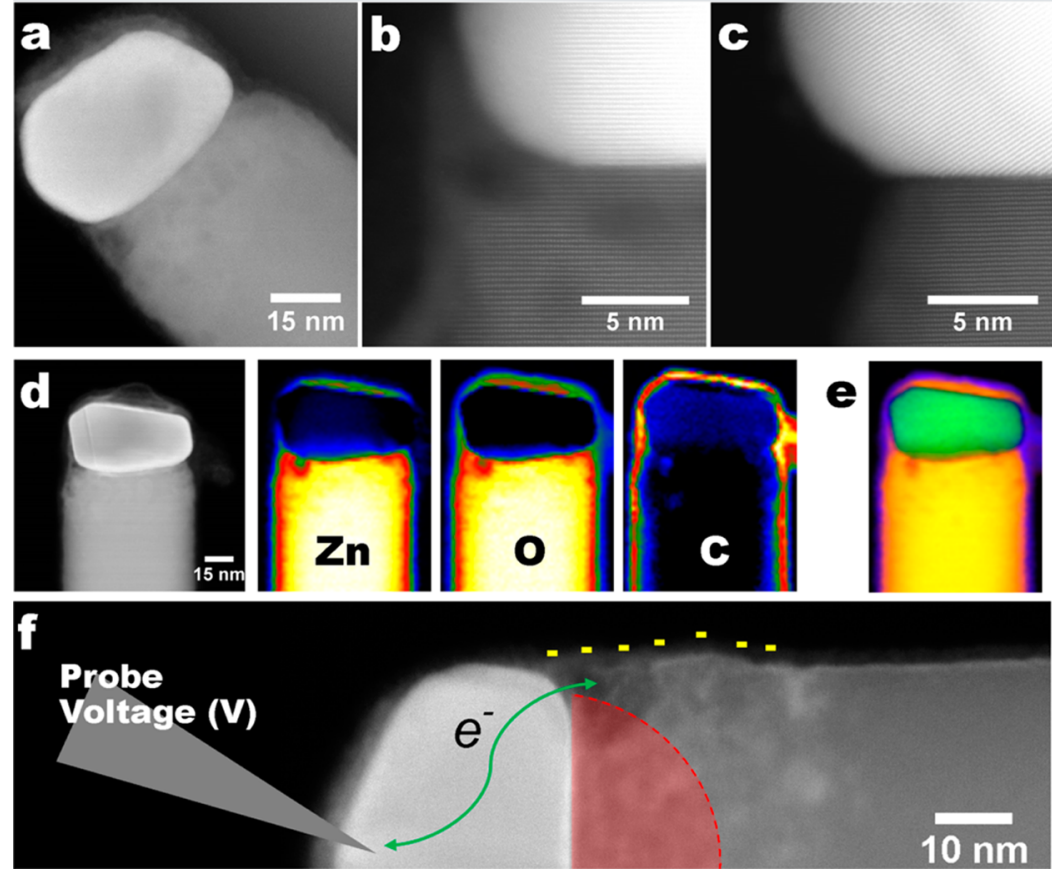

Figure 2. (a) MAADF-STEM image of a chronologically aged $\mathrm{ZnO}$ nanowire with Au nanocatalyst contact displaying the roughened neck and shell over the Au nanoparticle; (b) HAADF image of the edge region of the interface between a chronologically aged $\mathrm{ZnO}$ nanowire with Au nanocontact displaying the migration of $\mathrm{ZnO}$ at the contact edge; (c) HAADF image of a nonaged $\mathrm{ZnO}$ nanowire with no migration of $\mathrm{ZnO}$ at the contact edge; (d) MAADF image of a chronologically aged $\mathrm{ZnO}$ nanowire and the corresponding EELS maps of $\mathrm{Zn}\left(\mathrm{Zn}-\mathrm{L}_{2,3}\right), \mathrm{O}(\mathrm{O}-\mathrm{K})$, and $\mathrm{C}(\mathrm{C}-\mathrm{K})$, respectively, left to right; (e) overlay EELS map image of the data in (d), where $\mathrm{Zn}$ is red, O is yellow, $\mathrm{C}$ is blue, and the $\mathrm{Au}\left(\mathrm{Au}-\mathrm{M}_{4,5}\right)$ is shown as green; (f) diagram depicting a MAADF image that displays the defective neck region with increased electron concentration due to donor defects, Au contact, and the passage of charge at the edge of the Au-induced depletion region (red) during electrical measurement.

with the eSTEM measurements. Similar multiprobe measurements of vertical nonaged $\mathrm{ZnO}$ nanowires with $\mathrm{Au}$ nanocatalyst contacts have previously shown Schottky behavior when the $\mathrm{Au}$ particle is comparable in diameter to the nanowire (Figure S2c and accompanying text). ${ }^{7}$

To investigate this change in transport behavior, the same eSTEM nanowires were inspected with ac-STEM using highangle annular dark-field (HAADF), medium-angle annular dark-field (MAADF), and bright-field (BF) ac-STEM imaging (Figure 2). HAADF images give contrast dependent on highangle electron scattering, akin to Rutherford scattering, and their intensity therefore scales approximately as the square of the average atomic weight number of the imaged material (as $\sim Z^{1.7}$ ), such that HAADF is also known as "Z-contrast" imaging; MAADF images, while retaining Z-contrast characteristics, also contain contributions from diffraction contrast and are thus sensitive to surface and lattice strain. The MAADF image in Figure 2a displays the distinct $\mathrm{Au}$ nanoparticle and $\mathrm{ZnO}$ nanowire but also provides evidence for a distinct shell covering the $\mathrm{Au}$ particle as well as a surface roughness indicating diffusion of material at the $\mathrm{Au}-\mathrm{ZnO}$ neck. Figure $2 \mathrm{~b}$ shows an HAADF image of the $\mathrm{Au}$-nanowire interface edge region, with both sides of the interface aligned to a major crystallographic axis. Although less-prominent than in the strain-sensitive MAADF overview of Figure 2a, the "additional" diffuse material at the neck is clearly seen. ${ }^{26}$ Analysis of nonaged nanowires has shown a clean abrupt interface exists between the $\mathrm{Au}$ and nanowire, with little or no such material present at the neck or around the Au (Figure 2c; further images of nonaged nanowires are shown in Figure S3, and highresolution images in our previous works also provide further evidence). ${ }^{7,27}$ Electron energy-loss spectroscopy (EELS) mapping was employed in the ac-STEM (Figure $2 \mathrm{~d}$ ) to reveal that the shell and material at the neck consists of $\mathrm{Zn}$ and $\mathrm{O}$ with an additional carbon coating layer, likely present due to the lengthy storage period. An overlay image in Figure 2e includes the $\mathrm{Au}$ signal.

Over the extended storage time, the data on the aged nanowires shows that $\mathrm{ZnO}$ has migrated $\sim 20 \mathrm{~nm}$ from the nanowire surface neck region, coating the $\mathrm{Au}$ and leaving a pitted and defective $\mathrm{ZnO}$ surface. This microscopy analysis provides the first evidence of catalyst encapsulation by a strong metal-support interaction (SMSI) in a transition metal oxide$\mathrm{Au}$ system to occur in atmospheric conditions at room temperature and on relatively large metal nanoparticles (here, $30-50 \mathrm{~nm}) .^{28}$ SMSI, a well-known phenomenon in heterogeneous catalysis, is usually observed in reactive environments at elevated temperatures $\left(>200{ }^{\circ} \mathrm{C}\right)$ and is characterized by an encapsulation layer of substrate materials diffusing around the metal catalyst particle affecting the catalytic performance. ${ }^{28,29}$ Here, we have shown SMSI occurrings in $\mathrm{ZnO}$ nanowires with $\mathrm{Au}$ nanocatalyst growth particles at room temperature in ambient conditions over a time scale of years rather than minutes.

The interface between the $\mathrm{Au}$ and $\mathrm{ZnO}$ in the central region of the contact (observed here in projection; the roughened neck is found all around the nanowires) retained the high crystal quality and atomic abruptness that is apparent in nonaged $\mathrm{ZnO}$ nanowires with no diffusion of $\mathrm{Au}$ or blurring of the interface. The lack of diffusion across the $\mathrm{Au}-$ nanowire interface has been thoroughly investigated with through-focal sectioning that can reveal $\mathrm{Au}$ atoms on the surface or buried deep within the bulk. This is possible with aberration-corrected STEM instruments because of the large probe convergence 
angles (here, a $31 \mathrm{mrad}$ semiangle) that enable depth-slicing techniques, which exploit the reduced depth of field $(2-4 \mathrm{~nm})$ to locally image impurities, particularly at point defects. ${ }^{27,30}$ However, the crystal quality was degraded at the edge of the contact perimeter in all of the aged nanowires examined, and the $\mathrm{ZnO}$ at the contact interface edge region has lost the crystalline quality that is apparent immediately after growth (further images of the degraded interface edge, shell, and neck region are shown in Figure S4 with corresponding EELS maps).

Pristine nonaged $\mathrm{ZnO}$ nanowires with Au catalyst nanocontacts have been previously shown to produce a depletion region that is laterally constricted by the reduced interface size (typically $\sim 80 \%$ of nanowire diameter), an effect that was attributed to the necking that occurs at the edge of the contact interface. The effect of this laterally constricted depletion region are transport properties that are dominated by tunneling at the contact edge, where the depletion layer is thin. ${ }^{7}$ It can thus be expected that any structural or chemical modification of the necking region will have a large impact on the transport properties. In the case of the aged wires, the ac-STEM analysis showed the presence of an additional polycrystalline $\mathrm{ZnO}$ shell on the $\mathrm{Au}$ and $\mathrm{ZnO}$ material at the neck, which, in some instances, was also polycrystalline or held no obvious structure. MAADF images (Figures 2a,d,e and S4a) highlight the pitting that developed near the $\mathrm{Au}-\mathrm{ZnO}$ neck, which must create greater defect concentrations at the $\mathrm{ZnO}$ surface. High defect concentrations near metal contact interfaces to $\mathrm{ZnO}$ are known to create low-resistance ohmic Au contacts on single crystal ZnO. ${ }^{31,32}$ Furthermore, highly defective $\mathrm{ZnO}$ facets can predominantly exhibit donor defects such as zinc interstitials $\left(\mathrm{Zn}_{\mathrm{i}}\right)$ and oxygen vacancies $\left(\mathrm{V}_{\mathrm{O}}\right)^{32,33}$ that increase free electrons, and, coupled with SMSI-induced electron transfer ${ }^{28}$ from the $\mathrm{Au}$ contact, the electron concentration is locally increased near the interface on these highly defective and pitted $\mathrm{ZnO}$ neck and shell regions. The effect of this on the $\mathrm{Au}-\mathrm{ZnO}$ interface is to provide enhanced narrowing of the depletion region at the contact edge and an easy tunneling path through or around the Au-induced depletion region, as depicted in Figure $2 \mathrm{f}$. The easy tunneling is apparent in the $I-V$ measurements that all have near-linear transport behavior rather than the rectifying characteristics of nanowires in the initial nonaged state. The contribution of the amorphous carbon layer to the conduction at the surface is minimal, as current of only $\mathrm{nA}$ magnitude at $1 \mathrm{~V}$ was measured through the carbon shell (Figure S5), while the measurements of the nanowires here show current of $\sim \mu \mathrm{A}$. The linear $I-V$ behavior is consistent with previous finite-element transport simulations that showed rectifying $\mathrm{Au}$ nanocontacts on $\mathrm{ZnO}$ nanowires become ohmic when donor states, which create a surface carrier concentration of $1 \times 10^{13} \mathrm{~cm}^{-2}$, are present on the $\mathrm{ZnO}$ surface near the contact edge. ${ }^{7}$ This surface carrier concentration is considerably less than the highest theoretical and experimental values of $6.8 \times 10^{13} \mathrm{~cm}^{-2}$ and $8 \times 10^{13} \mathrm{~cm}^{-2}$, respectively. ${ }^{34,35}$

Examining the ac-STEM analysis of the same aged eSTEM nanowires revealed no obvious link between the $I-V$ characteristics and the nanowire diameter, $\mathrm{Au}$ diameter, and Au structure. A single nanowire was observed to have a stacking fault buried within the central region of the nanowire near the $\mathrm{Au}$ interface that might be expected to lead to potential barrier inhomogeneity affecting the transport behavior. ${ }^{36}$ However, the nanowire showed no unusual $I-V$ characteristics, which is entirely consistent with the model proposed here that describes the majority of the current flowing at the contact edge.
However, to confirm these conclusions and to directly compare the aged nanowires to nonaged nanowires, a freshly grown array was used to repeat the same eSTEM procedure. The freshly grown nanowires were transferred to the grid, screened with TEM, and then measured electrically before interrogation with ac-STEM and EELS analysis. The TEM and ac-STEM images clearly show that the nanowires lack the roughened neck region, and there is minimal diffusion of $\mathrm{Zn}$ around the Au particle (Figure $3 \mathrm{a}$ ). The $I-V$ characteristics of
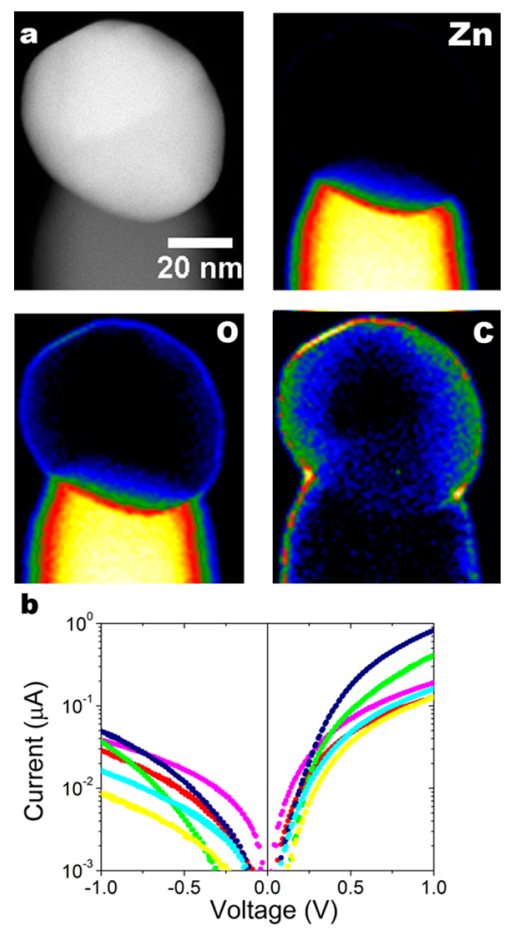

Figure 3. (a) HAADF image of a freshly grown $\mathrm{ZnO}$ nanowire and the corresponding EELS maps of $\mathrm{Zn}\left(\mathrm{Zn}-\mathrm{L}_{2,3}\right), \mathrm{C}(\mathrm{C}-\mathrm{K})$, and $\mathrm{O}(\mathrm{O}-\mathrm{K})$, respectively, in clockwise order; (b) a graph showing $I-V$ measurements of six nonaged nanowires in the eSTEM configuration, all exhibiting similar Schottky-like transport behavior.

the same nanowires show Schottky transport behavior (Figure $3 b)$, confirming the model of enhanced tunneling at the interface edge in the aged nanowires as a result of a SMSI. The ac-STEM analysis revealed that the $\mathrm{Au}-\mathrm{ZnO}$ interface of the freshly grown nanowires was lacking the diffuse material at the interface edge that is present on the aged nanowires. The EELS maps of the interface edge (Figure S6) comparing the aged nanowires to the freshly grown nanowires show that no $\mathrm{Zn}$ is present around the surface of $\mathrm{Au}$ particles on freshly grown nanowires, and no diffuse material is present at the interface edge; trace $\mathrm{O}$ present at the surface of the fresh wires is connected with the presence of hydrocarbon contamination and adsorption at the $\mathrm{Au}$ surface. Further EELS maps are presented in Figure S7. To illustrate the significant increase in the $\mathrm{Zn}$ and $\mathrm{O}$ signals that was measured on the aged nanowires at the Au surface, raw EELS spectra are shown in Figures S8 and 59 .

It is apparent from the above discussion that removal of the defective and diffuse material from the interface edge should eliminate the edge tunneling path of the aged nanowires. To test this hypothesis, nanowires were etched using DI water. The nanowires were initially cleaned with oxygen plasma $(5 \mathrm{~min}$, 


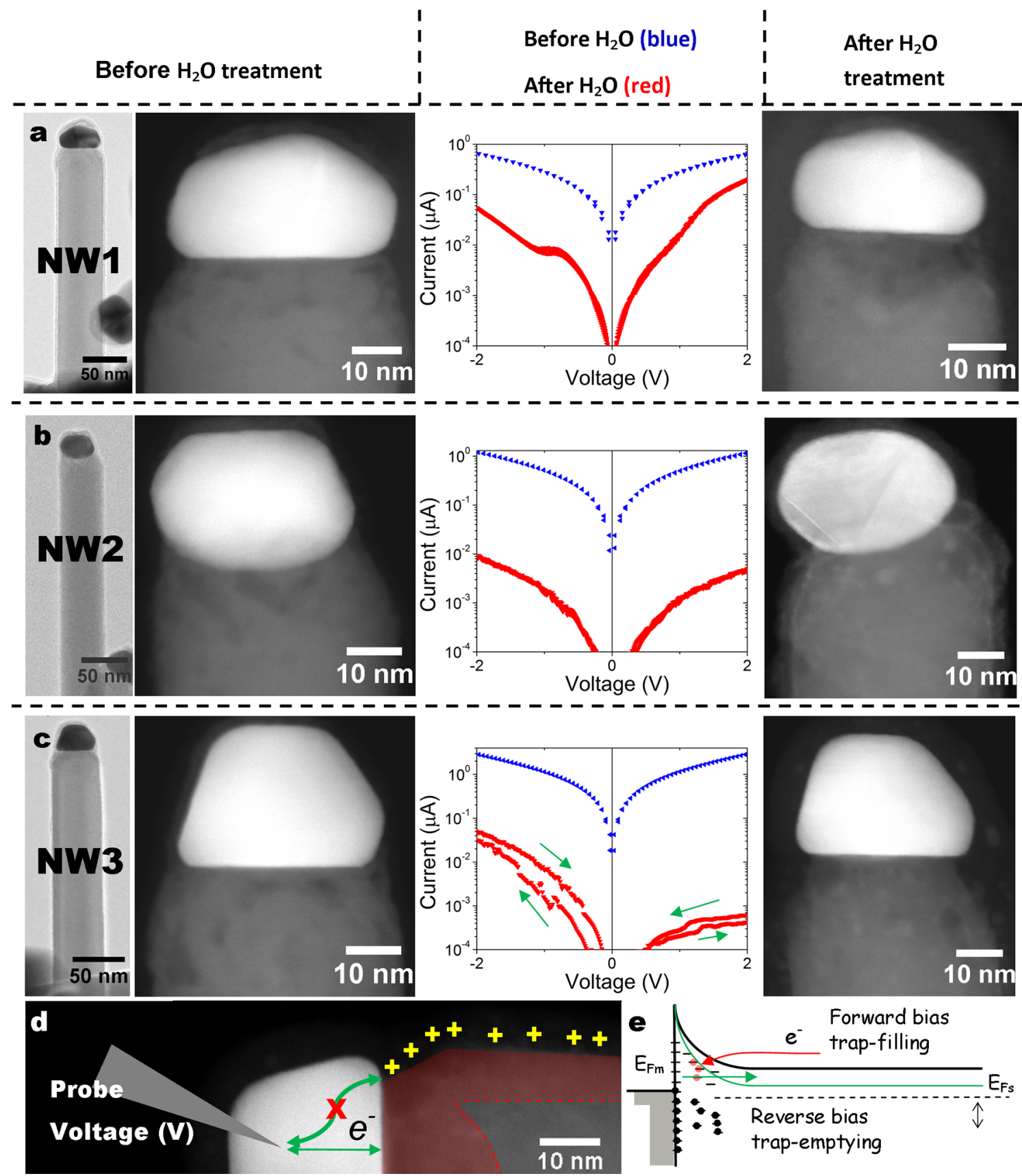

Figure 4. Examples of three $\mathrm{ZnO}$ nanowires with Au nanocontacts showing from left to right for (a) NW1, (b) NW2, and (c) NW3: bright-field TEM image of the complete nanostructure and Au nanoparticle at the tip in the eSTEM measurement configuration before measurement; HAADF ac-STEM image of the Au nanocontact and $\mathrm{ZnO}$ showing the chronologically degraded near-interface region corresponding to the blue $I-V$ data; $I-$ $V$ data before (blue) and after etching (red) with deionized $\mathrm{H}_{2} \mathrm{O}$; HAADF ac-STEM images after etching and electrical measurement corresponding to the red $I-V$ data, (d) diagram depicting a HAADF image of NW3 that displays the undercut neck region with acceptor defect-induced electron depletion (red), Au contact, and the passage of charge at the Au contact, and (e) schematic band diagram depicting the filling of trap states in forward-bias and the emptying of trap states in reverse-bias that becomes evident in the $I-V$ characteristics once the edge tunneling path is etched away.

$50 \mathrm{~W}, 0.2 \mathrm{mbar})$ to remove the carbon coat and allow uniform exposure to the immersion in deionized $\mathrm{H}_{2} \mathrm{O}(5 \mathrm{~min}, \mathrm{pH} \approx$ 5.5-6.0). ${ }^{37}$ The etching effect is attributed to the chemical disassociation

$$
\mathrm{ZnO}(\mathrm{s})+2 \mathrm{H}^{+} \rightarrow \mathrm{Zn}^{2+}+\mathrm{H}_{2} \mathrm{O}
$$

that leads to dissolution of surface $\mathrm{ZnO}$. The DI water treatment reduces the $\mathrm{ZnO}$ shell (Figures S8-S10) and of greatest consequence, results in preferential etching of the $\mathrm{ZnO}$ near the Au particle, undercutting the Au crystal to some extent (Figure S11) that removes the tunneling current path at the edge of the contact. The aqueous treatment also lightly etches the nanowire shaft, creating distinct roughness on some nanowires. $^{37}$

The $I-V$ behavior of the suspended eSTEM aged nanowires changed dramatically following the etching treatment, exhibiting a much-lower conductivity (reduction by a factor of $10^{-1}$ to $10^{-2} \mu \mathrm{A}$ at $\left.\pm 1 \mathrm{~V}\right)$. This change is a result of three factors: the 
reduced interface size, removal of the $\mathrm{ZnO}$ at the contact edge that leads to edge-tunneling, and increased nanowire roughness creating a greater concentration of acceptor defects from the exposed $\mathrm{O}^{2-}$ lattice sites after the dissolution of the lattice $\mathrm{Zn}^{2+}$ at or near the nanowire surface producing greater surface depletion. $^{38}$ The $I-V$ measurements after the etch displayed a range of characteristics from asymmetric to rectifying, reverse rectifying, and symmetrical " $\mathrm{S}$ "-shaped $I-V$ behavior that is usually found as a consequence of metal-semiconductormetal structures with back-to-back Schottky contacts. A results matrix correlating the electron microscopy of three nanowires to the eSTEM $I-V$ measurements is shown in Figure 4.

NW1 has changed from ohmic-like to rectifying after the $\mathrm{H}_{2} \mathrm{O}$ etch, and on close inspection of the microscopy images, it is apparent that the undercutting of the semiconductor has partially affected the $\mathrm{Au}$-nanowire interface with preferential etching occurring on the right side of the nanowire near the junction. Etching has concentrated near the Au interface, where a pit was originally observed after the chronological aging process (see Figure 4a and the MAADF images in Figure S12). The etching of the left side of the nanowire is only slight, but the removal of the $\mathrm{ZnO}$ at the contact edge region has removed the edge-tunneling path creating rectifying behavior. Furthermore, the variable degraded structure across the contact seemingly leads to NW1 showing characteristics of reversebias breakdown at $\sim-1 \mathrm{~V}$. The Au nanocontact on NW2 (Figure $4 \mathrm{~b}$ ) has been severely undercut, producing a much reduced connection to the nanowire after the etch; this accounts for the reduction in current by a factor of $\sim 10^{-2} \mu \mathrm{A}$. NW3 (Figure 4c) displayed uniform undercutting of the $\mathrm{ZnO}$ at the interface edge and a substantially degraded structure near the $\mathrm{Au}$ junction, producing unusual reverse rectifying behavior.

In the ideal case of a Schottky contact, reverse-bias current is negligible and is associated with tunneling and defect-related midgap states. ${ }^{10}$ Tunneling is the dominant transport mechanism in reverse bias because thermionic emission is negligible for the intrinsic barrier height $(\sim 0.6 \mathrm{eV})^{7,39}$ of $\mathrm{Au}-$ $\mathrm{ZnO}$ contacts and a greater concentration of defects will create midgap states. ${ }^{7} \mathrm{ZnO}$ is intrinsically defect-rich with a range of energy states within the bandgap, but these are not usually apparent in the $I-V$ characteristics of nonaged and chronologically aged nanowires because of the dominance of tunneling at the contact edge, where the depletion region is thin due to geometrical and surface effects that allow carriers to bypass the contact central zone. The etching here has removed the nanowire material that is responsible for the edge tunneling path (shown at the atomic scale in Figure S13) and enhanced the nanowire surface depletion layer. Consequently, carrier transport can only arise in the central depletion region reducing the measured current by several orders of magnitude, depicted in Figure 4d.

However, it is evident for NW3 that charge carrier tunneling through the depletion region is assisted by intrinsic or extrinsic defects, or both, near the interface that allow carriers to tunnel through defect energy states and more so in reverse-bias. This is apparent from the increased reverse-bias current relative to forward-bias current. In contrast, forward-bias transport is dominated by thermionic emission over the potential barrier that is limited after etching by the reduced interface size, reduced nanowire conductivity, and by an increased concentration of traps near the interface that are intrinsic and created by the degraded nature of the $\mathrm{Au}-$ nanowire junction. ${ }^{9,31}$ These ideas are summarized in the band diagram (Figure $3 \mathrm{e}$ ). The presence of defects are also evident from the hysteresis in the $I-V$ characteristics of NW3 that signifies the trapping and release of charge carriers in addition to the comparably greater conductivity in reverse-bias when tunneling has a greater effect due to the thinner depletion region and downward shift of the semiconductor Fermi level. The same change in $I-V$ characteristics was measured in the two-probe vertical configuration after the chronologically aged nanowire array was treated with the same plasma and etching process, confirming that the results from the eSTEM experiments shown in Figure 4 were not a consequence of the eSTEM experimental configuration (Figure S14 and description).

In summary, the measurements of the etched nanowires display that elimination of the edge-tunneling path unmasks the effect of defects on the transport characteristics and provides a method for simple modification of the near-interface nanowire surface that can be used to change the transport behavior of $\mathrm{Au}$-nanowire contacts. Simple surface modification through etching, deposition, or surface chemistry can thus potentially be used to create a complete range of contact transport characteristics with the same materials and interface qualities.

Conclusions. We have shown that the electrical transport properties of nanocontacts to nanowires are dominated by the structure of the semiconductor at the contact edge. Examining chronologically aged $\mathrm{ZnO}$ nanowires with $\mathrm{Au}$ nanocontacts displayed diffusion of $\mathrm{ZnO}$ around the $\mathrm{Au}$ particle due to a strong metal-support interaction creating a defective interface edge region and ohmic transport behavior. The importance of the edge region was further confirmed when the tunneling path at the contact perimeter was eliminated by removing material at the contact edge that changed the transport behavior from ohmic to a range of asymmetric, rectifying, reverse-rectifying, and symmetrical "S"-shaped $I-V$ characteristics. The susceptibility of the nanowires to etching under relatively benign liquid raises concerns over the application of the materials in future devices. However, the nanowires in all states are robust electrical components, and modifying the interface edge presents an opportunity to engineer the transport properties of nanowire contacts. The new concepts shown here provide interesting possibilities for transient electronics and reactive circuit breakers that require the material to diminish over time and eventually vanish.

Experimental Methods. Sample Preparation. $\mathrm{ZnO}$ nanowires were grown via a high-temperature vapor-phase method that used a thin $5 \mathrm{~nm}$ Au layer on the sapphire substrate to initiate growth and provide the one-step electrical nanocontacts on the nanowire tips. Lattice-matched sapphire (1120) was used to grow the nanowires in vertical arrays with single-crystal quality. ${ }^{16}$ To allow electrical probing of the nanowires and subsequent electron microscopy, the nanowires were mechanically transferred to the fingers of standard FIB lift-out copper grids (Omniprobe). The application of a standard TEM grid avoided restrictions on tilting degrees of freedom that some in situ transmission electron microscopy-scanning tunneling microscopy (TEM-STM) modules can present providing the distinct advantage that many nanowires could be aligned to a crystallographic axis, such that the metal-semiconductor junction was parallel to the beam. Fabrication of additional measurement contacts that are required for "on-chip" in situ measurement devices was not necessary, ensuring that the nanowire surface, nanowire structure, and $\mathrm{Au}-\mathrm{ZnO}$ interface were not unintentionally modified before measurement. Nanowires in the array configuration and on the grids were 
screened with a Hitachi S4800 SEM using the secondary electron and backscattered electron detectors. Nanowire grid samples were also screened with a Tecnai TF20 with brightfield imaging. The carbon surface layer described was removed using an oxygen plasma reactor for $5 \mathrm{~min}$ at $50 \mathrm{~W}$ and $0.2 \mathrm{mbar}$ pressure using a $99.99 \% \mathrm{O}_{2}$ source gas. Etching was performed after plasma cleaning by dipping the nanowires in deionized water for $5 \mathrm{~min}$ and then immediately vacuum-drying them. The extent of the etching was optimized on similar nanowires that were inspected with SEM.

Electrical Transport Measurement. The nanowires that were in the eSTEM cantilever configuration on the lift-out grids were loaded in the chamber of an UHV Omicron LT Nanoprobe. The tungsten STM probes that had been thermally annealed $^{17}$ were manually lowered to the height of the horizontal nanowires using the focal plane of the in situ SEM. With a small applied bias of $0.2 \mathrm{~V}$, one probe was brought into contact with the $\mathrm{Au}$ particle at the tip of the nanowire using real-time current feedback. The sample grid was at the low potential throughout the measurements. Once a signal above the noise was detected, the SEM beam was turned off, and $I-V$ sweeps were performed using a Keithley 2636 sourcemeter. $I-V$ sweeps using the two-probe method were performed in a similar fashion that has previously been described. ${ }^{7,18,25} I-V$ sweeps shown are an average of five measurements. The removal of probe oxides and contamination was confirmed by testing the resistance between the tips of two measurement probes $(\sim 350 \Omega)$ and between the probe and grid $(\sim 350 \Omega)$, which are comparable to the resistance of the measurement system $(\sim 150 \Omega)$.

Aberration-Corrected STEM. HAADF, MAADF, and BF imaging were carried out in a Nion UltraSTEM100 STEM operated at $100 \mathrm{keV}$ primary beam energy that was equipped with an UHV Enfina EELS spectrometer. The probe-forming optics, corrected for aberrations up to the fifth order, were configured to provide $\sim 50 \mathrm{pA}$ of beam current with a $31 \mathrm{mrad}$ beam convergence semiangle for an estimated probe size of 0.8 $\AA$. The inner and outer radii of the HAADF detector were calibrated at 79 and $195 \mathrm{mrad}$, respectively. Using this technique, nanowires were tilted to an available zone axis of the nanowire or Au particle, and the structure was imaged using phase contrast, MAADF, and HAADF imaging. To ensure minimal beam damage of the structures, core-loss EELS was carried out after the initial imaging and electrical assessment. Compositional EELS mapping was performed using the same beam configuration using $100 \mathrm{keV}$ primary beam energy and an exposure of $0.06 \mathrm{~s}$ per pixel. Chemical maps were created by integrating over a suitable energy window the intensity above the relevant EELS edges $\left(\mathrm{Zn}-\mathrm{L}_{2,3}, \mathrm{O}-\mathrm{K}, \mathrm{C}-\mathrm{K}\right.$, and $\left.\mathrm{Au}-\mathrm{M}_{4,5}\right)$ after removal of the decaying background using a power law model. The EELS data was systematically denoised using principal component analysis.

eSTEM procedure. The procedure followed these steps: (1) vertical nanowires from an array were mechanically transferred to the posts of a standard FIB lift-out grid; (2) the grid was loaded into the UHV multiprobe STM, and the probes were prepared by thermal annealing; (3) via in situ SEM, the measurement probe was contacted to the Au particle on the tip of a nanowire, and the reported electrical transport measurements were performed; (4) the grid was removed, and the same nanowires were studied with ac-STEM; (5) modification treatment was performed on the same nanowires, such as immersing in $\mathrm{H}_{2} \mathrm{O}$, and then step (3) was repeated in the UHV instrument; and (6) the same nanowires were then analyzed again with ac-STEM.

\section{ASSOCIATED CONTENT}

\section{Supporting Information}

The Supporting Information is available free of charge on the ACS Publications Web site at The Supporting Information is available free of charge on the ACS Publications website at DOI: 10.1021 /acs.nanolett.6b03699.

ac-STEM microscopy, EELS, and experimental I-V data and detailed descriptions of the experimental measurement process and nanowire properties. (PDF)

\section{AUTHOR INFORMATION}

\section{Corresponding Author}

*E-mail a.m.lord@swansea.ac.uk. ORCID

Alex M. Lord: 0000-0002-6258-2187

\section{Author Contributions}

The manuscript was written through contributions of all authors. All authors have given approval to the final version of the manuscript.

Notes

The authors declare no competing financial interest.

\section{ACKNOWLEDGMENTS}

This work was supported by the Centre for Nanohealth, Swansea University, UK. Aberration-corrected STEM analysis was performed at the SuperSTEM Laboratory, the UK National Facility for Aberration-Corrected STEM, funded by the EPSRC. Support was provided by the Engineering and Physical Sciences Research Council-funded Impact Acceleration Account (grant no. EP/K504002/1). TEM characterization data was enabled via support from the EPSRC-funded Leeds EPSRC Nanoscience and Nanotechnology Equipment Facility (LENNF) (grant no. EP/K023853/1).

\section{REFERENCES}

(1) No, Y.-S.; Gao, R.; Mankin, M. N.; Day, R. W.; Park, H.-G.; Lieber, C. M. Nano Lett. 2016, 16, 4713-4719.

(2) Webb, J. L.; Knutsson, J.; Hjort, M.; Ghalamestani, S. G.; Dick, K. A.; Timm, R.; Mikkelsen, A. Nano Lett. 2015, 15, 4865-4875.

(3) Kempa, T. J.; Kim, S.-K.; Day, R. W.; Park, H.-G.; Nocera, D. G.; Lieber, C. M. J. Am. Chem. Soc. 2013, 135, 18354-18357.

(4) Liu, Q.; Zou, R.; Wu, J.; Xu, K.; Lu, A.; Bando, Y.; Golberg, D.; Hu, J. Nano Lett. 2015, 15, 2809-2816.

(5) Fauske, V. T.; Huh, J.; Divitini, G.; Dheeraj, D. L.; Munshi, A. M.; Ducati, C.; Weman, H.; Fimland, B.-O.; van Helvoort, A. T. J. Nano Lett. 2016, 16, 3051-3057.

(6) Alam, S. B.; Panciera, F.; Hansen, O.; Mølhave, K.; Ross, F. M. Nano Lett. 2015, 15, 6535-6541.

(7) Lord, A. M.; Maffeis, T. G.; Kryvchenkova, O.; Cobley, R.; Kalna, K.; Kepaptsoglou, D. M. D.; Ramasse, Q. M.; Walton, A.; Ward, M. B.; Koeble, J.; Wilks, S. P. Nano Lett. 2015, 15, 4248-4254.

(8) Qin, W.; Hou, J.; Bonnell, D. A. Nano Lett. 2015, 15, 211-217.

(9) Yengui, M.; Riedel, D. J. Phys. Chem. C 2015, 119, 22700-22708.

(10) Rhoderick, E. H.; Williams, R. H. Metal-Semiconductor contacts; Clarendon Press: Oxford, UK, 1988.

(11) Suyatin, D. B.; Jain, V.; Nebol'sin, V. a; Tragardh, J.; Messing, M. E.; Wagner, J. B.; Persson, O.; Timm, R.; Mikkelsen, a; Maximov, I.; Samuelson, L.; Pettersson, H. Nat. Commun. 2014, 5, 3221.

(12) Léonard, F.; Talin, A.; Swartzentruber, B.; Picraux, S. Phys. Rev. Lett. 2009, 102, 106805. 
(13) Hui, H. Y.; Filler, M. A. Nano Lett. 2015, 15, 6939-6945.

(14) Gamalski, A. D.; Tersoff, J.; Kodambaka, S.; Zakharov, D. N.;

Ross, F. M.; Stach, E. A. Nano Lett. 2015, 15, 8211-8216.

(15) Fu, K. K.; Wang, Z.; Dai, J.; Carter, M.; Hu, L. Chem. Mater.

2016, 28, 3527-3539.

(16) Yang, P.; Yan, H.; Mao, S.; Russo, R.; Johnson, J.; Saykally, R.; Morris, N.; Pham, J.; He, R.; Choi, H.-J. Adv. Funct. Mater. 2002, 12, 323-331.

(17) Cobley, R. J.; Brown, R. a.; Barnett, C. J.; Maffeis, T. G. G.; Penny, M. W. Appl. Phys. Lett. 2013, 102, 023111.

(18) Lord, A. M.; Ward, M. B.; Evans, J. E.; Davies, P. R.; Smith, N. A.; Maffeis, T. G.; Wilks, S. P. J. Phys. Chem. C 2014, 118, 2117721184.

(19) Smith, N. A.; Lord, A. M.; Evans, J. E.; Barnett, C. J.; Cobley, R. J.; Wilks, S. P. Semicond. Sci. Technol. 2015, 30, 065011.

(20) Nelson, C. T.; Gao, P.; Jokisaari, J. R.; Heikes, C.; Adamo, C.; Melville, A.; Baek, S.-H.; Folkman, C. M.; Winchester, B.; Gu, Y.; Liu, Y.; Zhang, K.; Wang, E.; Li, J.; Chen, L.-Q.; Eom, C.-B.; Schlom, D. G.; Pan, X. Science 2011, 334, 968-971.

(21) Gao, P.; Nelson, C. T.; Jokisaari, J. R.; Baek, S.-H.; Bark, C. W.; Zhang, Y.; Wang, E.; Schlom, D. G.; Eom, C.-B.; Pan, X. Nat. Commun. 2011, 2, 591-596.

(22) Gao, P.; Nelson, C. T.; Jokisaari, J. R.; Zhang, Y.; Baek, S.-H.; Bark, C. W.; Wang, E.; Liu, Y.; Li, J.; Eom, C.-B.; Pan, X. Adv. Mater. 2012, 24, 1106-1110.

(23) Wang, L.; Zheng, K.; Zhang, Z.; Han, X. Nano Lett. 2011, 11, 2382-2385.

(24) Gan, Z.; Perea, D. E.; Yoo, J.; He, Y.; Colby, R. J.; Barker, J. E.; Gu, M.; Mao, S. X.; Wang, C.; Picraux, S. T.; Smith, D. J.; McCartney, M. R. J. Appl. Phys. 2016, 120, 104301.

(25) Lord, A. M.; Walton, A. S.; Maffeis, T. G.; Ward, M. B.; Davies, P.; Wilks, S. P. Nanotechnology 2014, 25, 425706.

(26) Hartel, P.; Rose, H.; Dinges, C. Ultramicroscopy 1996, 63, 93114.

(27) Lord, A. M.; Maffeis, T. G.; Walton, A. S.; Kepaptsoglou, D. M.; Ramasse, Q. M.; Ward, M. B.; Köble, J.; Wilks, S. P. Nanotechnology 2013, 24, 435706

(28) Liu, X.; Liu, M. H.; Luo, Y. C.; Mou, C. Y.; Lin, S. D.; Cheng, H.; Chen, J. M.; Lee, J. F.; Lin, T. S. J. Am. Chem. Soc. 2012, 134, 10251-10258.

(29) Zhang, S.; Plessow, P. N.; Willis, J. J.; Dai, S.; Xu, M.; Graham, G. W.; Cargnello, M.; Abild-Pedersen, F.; Pan, X. Nano Lett. 2016, 16, $4528-4534$.

(30) Van Benthem, K.; Lupini, A. R.; Oxley, M. P.; Findlay, S. D.; Allen, L. J.; Pennycook, S. J. Ultramicroscopy 2006, 106, 1062-1068.

(31) Mosbacker, H. L.; Zgrabik, C.; Hetzer, M. J.; Swain, A.; Look, D. C.; Cantwell, G.; Zhang, J.; Song, J. J.; Brillson, L. J. Appl. Phys. Lett. 2007, 91, 072102.

(32) Brillson, L. J.; Mosbacker, H. L.; Hetzer, M. J.; Strzhemechny, Y.; Jessen, G. H.; Look, D. C.; Cantwell, G.; Zhang, J.; Song, J. J. Appl. Phys. Lett. 2007, 90, 102116.

(33) Wang, Z.; Xue, J.; Han, D.; Gu, F. ACS Appl. Mater. Interfaces 2015, 7, 308-317.

(34) Moormann, H.; Kohl, D.; Heiland, G. Surf. Sci. 1980, 100, 302314.

(35) Li, H.; Bredas, J. L. Adv. Mater. 2016, 28, 3928-3936.

(36) Tung, R.; Levi, A.; Sullivan, J.; Schrey, F. Phys. Rev. Lett. 1991, $66,72-75$.

(37) Zhou, J.; Xu, N. S.; Wang, Z. L. Adv. Mater. 2006, 18, 24322435.

(38) Lord, A. M.; Maffeis, T. G.; Allen, M. W.; Morgan, D.; Davies, P. R.; Jones, D. R.; Evans, J. E.; Smith, N. A.; Wilks, S. P. Appl. Surf. Sci. 2014, 320, 664-669.

(39) Ozgur, U.; Alivov, Y. I.; Liu, C.; Teke, A.; Reshchikov, M. A.; Dogan, S.; Avrutin, V.; Cho, S.-J.; Morkoc, H. J. Appl. Phys. 2005, 98, 41301. 